\title{
Comparison of integrated PET/MRI with PET/CT in evaluation of endometrial cancer: a retrospective analysis of 81 cases
}

Li-hua Bian ${ }^{1}$, Min Wang ${ }^{1}$, Jing Gong ${ }^{1}$, Hong-hong Liu ${ }^{1}$, Nan Wang ${ }^{1}$, Na Wen ${ }^{1}$, Wen-sheng Fan ${ }^{1}$, Bai-xuan Xu ${ }^{1}$, Min-yang Wang ${ }^{1}$, Yuan-guang Meng ${ }^{\text {Corresp. } 1}$

${ }^{1}$ Department of Gynecology and Obstetrics, General Hospital of PLA, Beijing, China

Corresponding Author: Yuan-guang Meng

Email address: meng6512@vip.sina.com

Background: The objective of this study was to compare the diagnostic value of integrated PET/MRI with PET/CT for assessment of regional lymph node metastasis and deep myometrial invasion detection of endometrial cancer. Methods: Eighty-one patients with biopsy-proven endometrial cancer underwent preoperative PET/CT ( $n=37$ ) and integrated PET/MRI $(n=44)$ for initial staging. The diagnostic performance of PET/CT and integrated PET/MRI for assessing the extent of the primary tumor and metastasis to the regional lymph nodes was evaluated by two experienced readers. Histopathological and follow-up imaging results were used as the gold standard. McNemar's test was employed for statistical analysis. Results: Integrated PET/MRI and PET/CT both detected $100 \%$ of the primary tumors. Integrated PET/MRI proved significantly more sensitivity and specificity than PET/CT in regional lymph node metastasis detection $(P=0.015$ and $P<0.001$, respectively). The overall accuracy of myometrial invasion detection for PET/CT and Integrated PET/MRI was $45.9 \%$ and $81.8 \%$, respectively. Integrated PET/MRI proved significantly more accurate than PET/CT $(P<0.001)$. Conclusion: Integrated PET/MRI, which complements the individual advantages of MRI and PET, is a valuable technique for the assessment of the lymph node metastasis and myometrial invasion in patients with endometrial cancer. 
1 Comparison of Integrated PET/MRI with PET/CT in

2 Evaluation of Endometrial Cancer: A Retrospective

3 Analysis of 81 Cases

4

5 Li-hua Bian ${ }^{1}$, Min Wang ${ }^{1}$, Jing Gong ${ }^{1}$, Hong-hong Liu ${ }^{1}$, Nan Wang ${ }^{1}$, Na Wen ${ }^{1}$, Wen-sheng Fan ${ }^{1}$,

6 Bai-xuan $\mathrm{Xu}^{1}$, Min-yang Wang ${ }^{1}$, Yuan-guang Meng ${ }^{1}$

71 Department of Gynecology and Obstetrics, General Hospital of PLA, Beijing, People's

8 Republic of China.

9

10 Corresponding Author:

11 Yuan-guang Meng

12 Departments of Gynecology and Obstetrics, General Hospital of PLA, No. 28, Fuxing Rd, 13 Beijing 100853, People's Republic of China.

14 Email address: meng6512@vip.sina.com. 


\section{Abstract}

18 Background: The objective of this study was to compare the diagnostic value of integrated

19 PET/MRI with PET/CT for assessment of regional lymph node metastasis and deep myometrial

20 invasion detection of endometrial cancer.

21 Methods: Eighty-one patients with biopsy-proven endometrial cancer underwent preoperative

22 PET/CT $(\mathrm{n}=37)$ and integrated PET/MRI $(\mathrm{n}=44)$ for initial staging. The diagnostic

23 performance of PET/CT and integrated PET/MRI for assessing the extent of the primary tumor

24 and metastasis to the regional lymph nodes was evaluated by two experienced readers.

25 Histopathological and follow-up imaging results were used as the gold standard. McNemar's test 26 was employed for statistical analysis.

27 Results: Integrated PET/MRI and PET/CT both detected 100\% of the primary tumors. Integrated 28 PET/MRI proved significantly more sensitivity and specificity than PET/CT in regional lymph 29 node metastasis detection $(\mathrm{P}=0.015$ and $\mathrm{P}<0.001$, respectively). The overall accuracy of 30 myometrial invasion detection for PET/CT and Integrated PET/MRI was $45.9 \%$ and $81.8 \%$, 31 respectively. Integrated PET/MRI proved significantly more accurate than PET/CT $(\mathrm{P}<0.001)$.

32 Conclusion: Integrated PET/MRI, which complements the individual advantages of MRI and 33 PET, is a valuable technique for the assessment of the lymph node metastasis and myometrial 34 invasion in patients with endometrial cancer. 
36

37

38

39

40

41

42

43

44

\section{Introduction}

Endometrial cancer is the sixth most common cancer in women worldwide and the most common gynaecological malignancy in developed countries (Torre et al., 2015). The 2016 NCCN guidelines stated that the diagnosis and treatment of endometrial cancer should be referred to imaging studies (MRI/CT/PET), although it is still surgically staged (Koh et al., 2015). Accurate imaging diagnosis plays an important role in the treatment and prognosis of patients with endometrial cancer (Tsai et al., 2003).

PET/CT and PET/MRI have provided the basis for the comprehensive evaluation of patients with endometrial cancer before surgery, and are at present an important reference for the establishment of treatment plans (Kitajima et al., 2013). PET/CT has been reported to play a pivotal role in restaging field (Faria et al., 2015; Albano et al., 2019), lymph node metastasis and distant metastasis of endometrial cancer (Gee et al., 2018). However, due to the low resolution of soft tissue in PET/CT, it is less effective than MRI at detecting the myometrial invasion of endometrial cancer (Gallego et al., 2014; Kakhki et al., 2013). This classification is important for the treatment of endometrial cancer (Suri \& Arora, 2015). In addition, PET/CT radiation is also harmful to the patient. PET/MRI, as a multimodal molecular imaging technology, successfully integrates two imaging technologies: positron emission tomography and magnetic resonance imaging. PET/MRI, which combines the high precision of MRI and the high sensitivity of PET, has been shown to be valuable for the tumor size evaluation of endometrial cancer and lymph node metastasis (Kitajima et al., 2009; Queiroz et al., 2015; Stecco et al., 2016). PET/MRI currently has both integrated and sequential acquisition of PET and MRI images. However, most of the current research uses the method of post-fusion of PET and MRI images, and it is difficult 
58 to simultaneously acquire accurate PET and MRI images. The artifacts in fused image seriously

59 degrade the imagine quality and affect the exact diagnosis.

60 There are few studies on the evaluation of endometrial cancer using integrated PET/MRI.

61 The purpose of this report is to compare the evaluation efficacy of integrated PET/MRI and

$62 \mathrm{PET} / \mathrm{CT}$ on the diagnosis of endometrial cancer.

\section{Materials \& Methods}

\section{Patients}

65 This retrospective study was approved by the institutional review board, and the need for

66 patient informed consent was waived (Ethical Application Ref: GH301-18052). Our primary

67 patient selection criteria was pathologically proven diagnoses of endometrial cancer patients who

68 underwent pretreatment ${ }^{18} \mathrm{~F}-\mathrm{FDG}$ PET/CT or integrated ${ }^{18} \mathrm{~F}-\mathrm{FDG}$ PET/MRI for initial staging

69 between April 2013 and May 2018. According to the primary criteria, 81 consecutive patients

70 were selected. Of these, 37 patients underwent the ${ }^{18} \mathrm{~F}-\mathrm{FDG}$ PET/CT scanning, and 44 patients

71 underwent the integrated ${ }^{18}$ F-FDG PET/MRI scanning.

\section{PET/CT}

73 Whole body PET/CT images were obtained using a PET/CT scanner Biograph 64 PET/CT

74 (Siemens Healthcare Sector, Erlangen, Germany) as previously reported (Stecco et al., 2016).

75 Patients fasted for at least $6 \mathrm{~h}$ before tracer injection. After injection of 2.22 4.44 MBq/kg of $76{ }^{18}$ F-FDG, PET images were obtained after an approximately 60 -min uptake period with the 77 patient's arms raised to cover the orbitomeatal line to the proximal third of the femurs. After 78 obtaining a scout view (120-140 kVp, $30 \mathrm{mAs})$, the PET protocol comprised five to six bed 79 positions (3 min each) depending on the patients height. Three-dimensional image 
80 reconstructions were acquired using the iterative reconstruction algorithms. The duration of

$81 \mathrm{PET} / \mathrm{CT}$ acquisition was approximately $20 \mathrm{~min}$.

\section{Integrated PET/MRI}

83 Simultaneous PET/MRI images were obtained using an integrated PET/MRI scanner 84 BiographmMR (Siemens Healthcare Sector, Erlangen, Germany) as previously reported 85 (Schwartz et al., 2018). Patients fasted for at least $6 \mathrm{~h}$ before tracer injection. After injection of 2.22 4.44 MBq/kg of ${ }^{18} \mathrm{~F}-\mathrm{FDG}$, simultaneous PET /MRI scan was conducted. A coronal 3D Dixon volumetric interpolated breath- hold examination (VIBE) T1-weighted imaging sequence

88 in- and out-of -phase was acquired as a template for attenuation correction (AC). The Dixon MR acquisition, used for fat-water separation, was then segmented into four distinct tissue classifications for the MR- based AC map. The following additional MR sequences were used: half-Fourier acquire single-shot turbo spin echo (HASTE) T2 weighted imaging (axial and coronal); axial, coronal and sagittal T2; axial T2 Turbo spin echo (TSE) fat suppressed; diffusion weighted imaging (DWI) using various $b$ values $\left(50 / 800 \mathrm{~s} / \mathrm{mm}^{3}\right) ; 3 \mathrm{D}$ axial in and out of phase; axial VIBE pre- and post- contrast and sagittal VIBE post- contrast. PET acquisition was simultaneous with MR acquisition. PET protocol comprised five to six bed positions (5 min each). No gadolinium contrast was used for the MRI portion of the study. The PET/MRI examination lasted approximately $60 \mathrm{~min}$.

\section{Image analysis and standard of reference}

Two radiologists/nuclear medicine physicians (five years of experience working in PET/CT and PET/MRI) who were especially experienced in gynecological imaging, consensually and retrospectively evaluated PET/CT and Integrated PET/MRI images for the following findings: (a) presence of the primary tumor, (b) tumor extension into the myometrium, cervical stroma, 
103 uterine serosa or adnexa, vagina or parametrium, and urinary bladder or rectum mucosa as well

104 as (c) pelvic lymph nodes (Kitajima et al., 2013). Neither reader was aware of the results of other

105 imaging studies, histopathologic findings or clinical data.

106 Histopathological correlation was available in all 81 patients and was used as the reference 107 standard.

108 Statistical analysis

109 All statistical analyses were performed using IBM SPSS version 23.0 (SPSS Inc, Armonk,

$110 \mathrm{NY}$ ). The McNemar test was used to determine the statistical significance of differences in the

111 accuracy of staging as determined by PET/CT and Integrated PET/MRI. Compare and analyze

112 the data by the means of Frequencies, Crosstabs and Chi square test. Differences at $\mathrm{P}<0.05$ were

113 considered to be statistically significant.

114 Results

115 PET/CT and Integrated PET/MRI examinations were successfully completed in 37 patients 116 and 44 patients, respectively (Table 1). According to the revised International Federation of 117 Gynecology and Obstetrics (FIGO) criteria (Kitajima et al., 2009), the T stage was classified as 118 pT1a in 18 patients (PET/CT) and 24 patients (Integrated PET/MRI), pT1b in 5 (PET/CT) and 4 119 (Integrated PET/MRI), pT2 in 5 (PET/CT) and 2 (Integrated PET/MRI), pT3 in 2 (PET/CT) and 1206 (Integrated PET/MRI), and pT4 in 1 (PET/CT) and 0 (Integrated PET/MRI). The 121 histopathologic types of the primary tumors were Adenocarcinoma: Grade 1 (14 in PET/CT, and 12213 in Integrated PET/MRI), Grade 2 (16 in PET/CT, and 23 in Integrated PET/MRI), and Grade 1233 (7 in PET/CT, and 8 in Integrated PET/MRI). Demographic data for the 81 patients are shown 124 in Table 1 and Table 2.

125 Primary tumor detection 
Both PET/CT and Integrated PET/MRI detected 100\% of the primary tumors (Table 3 ).

127

128

129

130

131

132

133

134

135

136

137

138

139

140

141

142

143

144

145

146

147 Two representative cases are shown (Figure 1).

\section{Discussion \\ Discussion} than PET/CT $(\mathrm{P}=0.015$ and $\mathrm{P}<0.001$, respectively $)$. PET/MRI proved more specificity than PET/CT.

\section{Deep myometrial invasion detection}

\section{Regional lymph node metastasis and abdominal metastasis detection}

The overall accuracy of regional lymph node metastasis detection for PET/CT and Integrated PET/MRI was $86.5 \%$ and $95.5 \%$, respectively (Table 3). Of 3 positive pelvic lymph nodes, PET/CT correctly identified 1 as positive, with 2 false negative lesions, resulting in a sensitivity per lesion of $33.3 \%$. Of 4 positive pelvic lymph nodes, Integrated PET/MRI correctly identified 2 as positive, with 2 false negative lesions, resulting in a sensitivity per lesion of 50.0\%. Integrated PET/MRI proved more accurate than PET/CT, although the difference was not significant $(\mathrm{P}=0.113)$. Integrated $\mathrm{PET} / \mathrm{MRI}$ proved significantly more sensitivity and specificity

The overall accuracy of abdominal metastasis detection for PET/CT and Integrated PET/MRI was $97.3 \%$ and $100 \%$, respectively (Table 3). When correlated with histopathology, the only one false positive of PET/CT was liver metastasis. Integrated PET/MRI proved more accurate than $\mathrm{PET} / \mathrm{CT}$, although the difference was not significant $(\mathrm{P}=0.081)$. Integrated

PET/CT over-staged the myometrial invasion in 6 patients $(16.2 \%)$ and under-staged it in 11 patients (29.7\%). Integrated PET/MRI over-staged the myometrial invasion in 3 patients $(6.8 \%)$ and under-staged it in5 patients $(11.4 \%)$. The overall accuracy of myometrial invasion detection for PET/CT and Integrated PET/MRI was 45.9\% and 81.8\%, respectively (Table 4). Integrated PET/MRI proved significantly more accurate than PET/CT $(\mathrm{P}<0.001)$. 
This retrospective analysis compared the efficacy of PET/CT and integrated PET/MRI in the 150 staging of endometrial cancer. PET/CT and integrated PET/MRI are similar in the diagnostic 151 efficacy of endometrial cancer and its local lymph node metastasis. As expected, integrated

152 PET/MRI is more sensitive to the diagnosis of myometrial invasion of endometrial cancer than $153 \mathrm{PET} / \mathrm{CT}$.

154 Traditional PET/CT and fused PET/MRI have been studied for tumor detection and 155 lymphatic metastasis of endometrial cancer (Kitajima et al., 2013; Queiroz et al., 2015; Stecco et 156 al., 2016). Kitajima et al. found that fused PET/MRI is better than MRI at diagnosing lymph 157 node metastasis of endometrial cancer (100\% vs. 66.7\%) (Kitajima et al., 2013). Stecco et al. 158 reported that fused PET/MRI had more sensitivity, specificity and diagnostic accuracy than 159 PET/CT for the diagnosis of lymph node metastasis (Stecco et al., 2016). Similar to their results, 160 our study demonstrated that integrated PET/MRI has an advantage over PET/CT in sensitivity. 161 However, the integrated PET/MRI and PET/CT showed a consistent $100 \%$ diagnostic accuracy 162 for tumors.

163 MRI is very advantageous for diagnosing localized tumors, especially high-risk factors such 164 as tumor size, myometrial invasion, and cervical infiltration. Therefore, using the advantages of 165 MRI, integrated PET/MRI is more advantageous than PET/CT for diagnosing myometrial 166 invasion of endometrial cancer (Sawicki et al., 2018; Nakajo et al., 2010). Our results provide 167 proof. Our data are also consistent with Duncan's results (Duncan et al., 2012), which is obtained 168 using fused PET/MRI. Kitajima's study also found that fused PET/MRI is better than PET/CT 169 for the diagnosis of endometrial cancer (80\% vs. 60\%) (Kitajima et al., 2013). Since integrated $170 \mathrm{PET} / \mathrm{MRI}$ is simultaneous, its scanning time is also less than fused PET/MRI. In addition, the 171 Integrated PET/MRI presents several challenges, including high costs of acquisition and 
172 screening, lack of standardized imaging protocols, and limitations in patients with peacemakers

173 or claustrophobia (Bailey et al., 2015; Wehrl et al., 2010).

174 Increasing evidence supports the role of sentinel lymph node mapping (SLNM) for

175 endometrial cancer (Rossi et al., 2017). PET/CT also appeared to improve sentinel lymph node

176 detection in cervical and uterine cancer (Pandit-Taskar et al., 2010). Considering the higher

177 accuracy of integrated PET-MRI for regional lymph node metastasis detection, we concluded

178 that the combination of integrated PET-MRI and sentinel lymph node mapping is reasonable and

179 feasible.

180 Limitations

181 This study has several limitations. First, we did not allow patients to simultaneously test the

$182 \mathrm{PET} / \mathrm{CT}$ and integrated PET/MRI, but only randomly asked them to assess one method, due to 183 patient compliance and economic reasons. This may increase the test's interference factor.

184 Furthermore, this study is a retrospective analysis, and the number of patients according to the 185 inclusion criteria is relatively small. Thus, our conclusions can only be considered preliminary. It 186 is imperative to study the differences in the diagnosis of endometrial cancer between PET/CT 187 and integrated PET/MRI using large-scale prospective clinical trials.

188 Conclusions

189 Integrated PET/MRI imaging showed a higher application value for the diagnosis and 190 staging of endometrial cancer diseases, but more studies are necessary to investigate its potential 191 clinical utility.

192 Acknowledgements

$193 \quad$ None.

194 References 
Albano, D., Zizioli, V., Odicino, F., Giubbini, R., and Bertagna, F. 2019. Clinical and prognostic value of (18)FFDG PET/CT in recurrent endometrial carcinoma. Rev Esp Med Nucl Imagen Mol 38:87-93. 10.1016/j.remn.2018.09.005

Bailey, D.L., Antoch, G., Bartenstein, P., Barthel, H., Beer, A.J., Bisdas, S., Bluemke, D.A., Boellaard, R., Claussen, C.D., Franzius, C., Hacker, M., Hricak, H., la Fougere, C., Guckel, B., Nekolla, S.G., Pichler, B.J., Purz, S., Quick, H.H., Sabri, O., Sattler, B., Schafer, J., Schmidt, H., van den Hoff, J., Voss, S., Weber, W., Wehrl, H.F., and Beyer, T. 2015. Combined PET/MR: The Real Work Has Just Started. Summary Report of the Third International Workshop on PET/MR Imaging; February 17-21, 2014, Tubingen, Germany. MOLECULAR IMAGING AND BIOLOGY 17:297-312. 10.1007/s11307-014-0818-0

Duncan, K.A., Drinkwater, K.J., Frost, C., Remedios, D., and Barter, S. 2012. Staging cancer of the uterus: a national audit of MRI accuracy. CLINICAL RADIOLOGY 67:523-530. 10.1016/j.crad.2011.10.019

Faria, S.C., Sagebiel, T., Balachandran, A., Devine, C., Lal, C., and Bhosale, P.R. 2015. Imaging in endometrial carcinoma. Indian J Radiol Imaging 25:137-147. 10.4103/0971-3026.155857

Gallego, J.C., Porta, A., Pardo, M.C., and Fernandez, C. 2014. Evaluation of myometrial invasion in endometrial cancer: comparison of diffusion-weighted magnetic resonance and intraoperative frozen sections. $A B D O M I N A L$ IMAGING 39:1021-1026. 10.1007/s00261-014-0134-9

Gee, M.S., Atri, M., Bandos, A.I., Mannel, R.S., Gold, M.A., and Lee, S.I. 2018. Identification of Distant Metastatic Disease in Uterine Cervical and Endometrial Cancers with FDG PET/CT: Analysis from the ACRIN 6671/GOG 0233 Multicenter Trial. RADIOLOGY 287:176-184. 10.1148/radiol.2017170963

Kakhki, V.R., Shahriari, S., Treglia, G., Hasanzadeh, M., Zakavi, S.R., Yousefi, Z., Kadkhodayan, S., and Sadeghi, R. 2013. Diagnostic performance of fluorine 18 fluorodeoxyglucose positron emission tomography imaging for detection of primary lesion and staging of endometrial cancer patients: systematic review and meta-analysis of the literature. INTERNATIONAL JOURNAL OF GYNECOLOGICAL CANCER 23:1536-1543. 10.1097/IGC.0000000000000003

Kitajima, K., Murakami, K., Yamasaki, E., Kaji, Y., and Sugimura, K. 2009. Accuracy of integrated FDG$\mathrm{PET} /$ contrast-enhanced $\mathrm{CT}$ in detecting pelvic and paraaortic lymph node metastasis in patients with uterine cancer. EUROPEAN RADIOLOGY 19:1529-1536. 10.1007/s00330-008-1271-8

Kitajima, K., Suenaga, Y., Ueno, Y., Kanda, T., Maeda, T., Takahashi, S., Ebina, Y., Miyahara, Y., Yamada, H., and Sugimura, K. 2013. Value of fusion of PET and MRI for staging of endometrial cancer: comparison with (1)(8)FFDG contrast-enhanced PET/CT and dynamic contrast-enhanced pelvic MRI. EUROPEAN JOURNAL OF RADIOLOGY 82:1672-1676. 10.1016/j.ejrad.2013.05.005

Koh, W.J., Greer, B.E., Abu-Rustum, N.R., Apte, S.M., Campos, S.M., Cho, K.R., Chu, C., Cohn, D., Crispens, M.A., Dizon, D.S., Dorigo, O., Eifel, P.J., Fisher, C.M., Frederick, P., Gaffney, D.K., George, S., Han, E., Higgins, S., Huh, W.K., Lurain, J.R., Mariani, A., Mutch, D., Fader, A.N., Remmenga, S.W., Reynolds, R.K., Tillmanns, T., Valea, F.A., Yashar, C.M., McMillian, N.R., and Scavone, J.L. 2015. Uterine Sarcoma, Version 1.2016: Featured Updates to the NCCN Guidelines. J Natl Compr Canc Netw 13:1321-1331.

Nakajo, K., Tatsumi, M., Inoue, A., Isohashi, K., Higuchi, I., Kato, H., Imaizumi, M., Enomoto, T., Shimosegawa, E., Kimura, T., and Hatazawa, J. 2010. Diagnostic performance of fluorodeoxyglucose positron emission tomography/magnetic resonance imaging fusion images of gynecological malignant tumors: comparison with positron emission tomography/computed tomography. Japanese Journal of Radiology 28:95-100. 10.1007/s11604009-0387-3

Pandit-Taskar, N., Gemignani, M.L., Lyall, A., Larson, S.M., Barakat, R.R., and Abu, R.N. 2010. Single photon emission computed tomography SPECT-CT improves sentinel node detection and localization in cervical and uterine malignancy. GYNECOLOGIC ONCOLOGY 117:59-64. 10.1016/j.ygyno.2009.12.021

Queiroz, M.A., Kubik-Huch, R.A., Hauser, N., Freiwald-Chilla, B., von Schulthess, G., Froehlich, J.M., and VeitHaibach, P. 2015. PET/MRI and PET/CT in advanced gynaecological tumours: initial experience and comparison. EUROPEAN RADIOLOGY 25:2222-2230. 10.1007/s00330-015-3657-8

Rossi, E.C., Kowalski, L.D., Scalici, J., Cantrell, L., Schuler, K., Hanna, R.K., Method, M., Ade, M., Ivanova, A., and Boggess, J.F. 2017. A comparison of sentinel lymph node biopsy to lymphadenectomy for endometrial cancer staging (FIRES trial): a multicentre, prospective, cohort study. LANCET ONCOLOGY 18:384-392. 10.1016/S14702045(17)30068-2

Sawicki, L.M., Kirchner, J., Grueneisen, J., Ruhlmann, V., Aktas, B., Schaarschmidt, B.M., Forsting, M., Herrmann, K., Antoch, G., and Umutlu, L. 2018. Comparison of (18)F-FDG PET/MRI and MRI alone for whole-body staging 
250

251

252

253

254

255

256

257

258

259

260

261

262

263

264

265

266

267 and potential impact on therapeutic management of women with suspected recurrent pelvic cancer: a follow-up study. Eur J Nucl Med Mol Imaging 45:622-629. 10.1007/s00259-017-3881-3

Schwartz, M., Gavane, S.C., Bou-Ayache, J., Kolev, V., Zakashansky, K., Prasad-Hayes, M., Taouli, B., Chuang, L., and Kostakoglu, L. 2018. Feasibility and diagnostic performance of hybrid PET/MRI compared with PET/CT for gynecological malignancies: a prospective pilot study. Abdom Radiol (NY). 10.1007/s00261-018-1665-2

Stecco, A., Buemi, F., Cassara, A., Matheoud, R., Sacchetti, G.M., Arnulfo, A., Brambilla, M., and Carriero, A. 2016. Comparison of retrospective PET and MRI-DWI (PET/MRI-DWI) image fusion with PET/CT and MRI-DWI in detection of cervical and endometrial cancer lymph node metastases. Radiologia Medica 121:537-545. 10.1007/s11547-016-0626-5

Suri, V., and Arora, A. 2015. Management of Endometrial Cancer: A Review. Rev Recent Clin Trials 10:309-316.

Torre, L.A., Bray, F., Siegel, R.L., Ferlay, J., Lortet-Tieulent, J., and Jemal, A. 2015. Global cancer statistics, 2012. CA Cancer J Clin 65:87-108. 10.3322/caac.21262

Tsai, C.C., Tsai, C.S., Ng, K.K., Lai, C.H., Hsueh, S., Kao, P.F., Chang, T.C., Hong, J.H., and Yen, T.C. 2003. The impact of image fusion in resolving discrepant findings between FDG-PET and MRI/CT in patients with gynaecological cancers. Eur J Nucl Med Mol Imaging 30:1674-1683. 10.1007/s00259-003-1300-4

Wehrl, H.F., Sauter, A.W., Judenhofer, M.S., and Pichler, B.J. 2010. Combined PET/MR imaging--technology and applications. Technol Cancer Res Treat 9:5-20. 10.1177/153303461000900102 


\section{Figure 1}

Two representative cases $\square$

Patient 1: A 60-year-old woman with endometrial cancer and pelvic lymph node metastases.

Axial PET/CT shows intense ${ }^{18} \mathrm{~F}-\mathrm{FDG}$ uptake by uterine cavity (black arrow) and lymph nodes (white arrow), suggesting malignancy. Patient 2: A 55-year-old woman with endometrial cancer and pelvic lymph node metastases. Axial Integrated PET/MRI shows intense ${ }^{18} \mathrm{~F}$-FDG uptake by uterine cavity (black arrow) and lymph nodes (white arrow). Histopathologic examination confirmed cancer involvement in these lymph nodes.
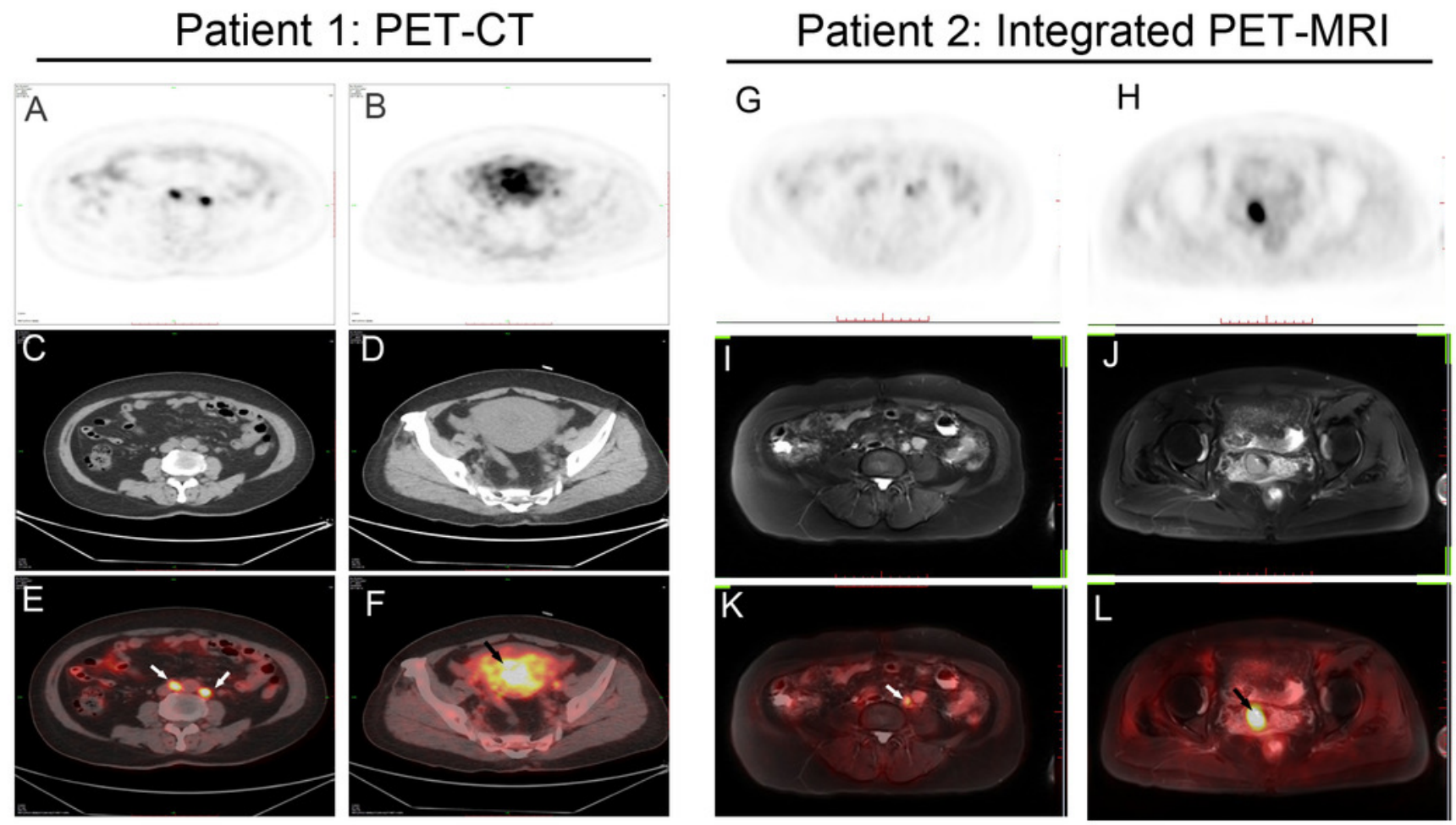
Table $\mathbf{1}$ (on next page)

Demographic and clinical characteristics of the study population 
1 Table 1 Demographic and clinical characteristics of the study population

\begin{tabular}{llll}
\hline & PET/CT & $\begin{array}{l}\text { Integrated } \\
\text { PET/MRI }\end{array}$ & p value \\
\hline Number of patients & 37 & 44 & $/$ \\
Age in years, mean (range) & $53.2(28-76)$ & $54.2(35-73)$ & 0.59 \\
BMI & 26.2 & 24.5 & 0.23 \\
Indication, number (\%) & & & \\
$\quad$ Staging & 37 & 44 & $/$ \\
$\quad$ Re-staging & 0 & 0 & 0.28 \\
Treatment, number (\%) & & & \\
$\quad$ - Surgery & 100 & 100 & \\
$\quad$ Surgery only (or curettage) & 21.6 & 36.4 & \\
$\quad$ With additional chemotherapy & 8.1 & 13.6 & \\
$\quad$ With additional progesterone & 48.7 & 38.6 & \\
$\quad$ With additional radiotherapy & 21.6 & 11.4 & \\
- No surgery & 0 & 0 & \\
- No treatment & 0 & 0 & 0.84 \\
$\quad$ - Dead before treatment & 0 & 0 & \\
Tumor size (cm) & $0.6-6.5$ & $0.7-7.1$ & \\
\hline
\end{tabular}




\section{Table 2 (on next page)}

Frequency and percentage of FIGO classification and tumor histotype of the study population 
1 Table 2 Frequency and percentage of FIGO classification and tumor histotype of the study population

\begin{tabular}{llll}
\hline & PET/CT & $\begin{array}{l}\text { Integrated } \\
\text { PET/MRI }\end{array}$ & $\boldsymbol{p}$ value \\
\hline FIGO stage & 24 & 32 & 0.29 \\
IA & 5 & 4 & \\
IB & 5 & 2 & \\
II & 0 & 3 & \\
IIIA & 0 & 0 & \\
IIIB & 2 & 2 & \\
IIIC1 & 0 & 1 & \\
IIIC2 & 1 & 0 & \\
IV & & & \\
Frequency of endometrium cancer & 14 & 13 & \\
Adenocarcinoma G1 & 16 & 23 & \\
Adenocarcinoma G2 & 7 & 8 & \\
Adenocarcinoma G3 & &
\end{tabular}

2 


\section{Table 3(on next page)}

Parameters of diagnostic performance including FP, FN, TP, TN, sensitivity, specificity. Accuracy, PPV, and NPV of PET/CT and Integrated PET/MRI on a per-patient basis 
1 Table 3 Parameters of diagnostic performance including FP, FN, TP, TN, sensitivity, specificity. Accuracy,

2 PPV, and NPV of PET/CT and Integrated PET/MRI on a per-patient basis

\begin{tabular}{ccccccc}
\hline & \multicolumn{2}{c}{$\begin{array}{c}\text { Primary tumor } \\
\text { detection }\end{array}$} & \multicolumn{2}{c}{$\begin{array}{c}\text { Regional lymph } \\
\text { node metastasis }\end{array}$} & \multicolumn{2}{c}{$\begin{array}{c}\text { Abdominal } \\
\text { metastasis }\end{array}$} \\
\cline { 2 - 7 } & PET/CT & $\begin{array}{c}\text { Integrated } \\
\text { PET/MRI }\end{array}$ & PET/CT & $\begin{array}{c}\text { Integrated } \\
\text { PET/MRI }\end{array}$ & PET/CT & $\begin{array}{c}\text { Integrated } \\
\text { PET/MRI }\end{array}$ \\
\hline TP & 37 & 44 & 1 & 2 & 0 & 0 \\
TN & 0 & 0 & 31 & 40 & 36 & 44 \\
FP & 0 & 0 & 3 & 0 & 1 & 0 \\
FN & 0 & 0 & 2 & 2 & 0 & 0 \\
Sensitivity & 100 & 100 & 33.3 & 50 & 100 & 100 \\
Specificity & 100 & 100 & 91.2 & 100 & 97.3 & 100 \\
PPV & 100 & 100 & 75 & 100 & 100 & 100 \\
NPV & 100 & 100 & 93.9 & 95.2 & 100 & 100 \\
Accuracy & 100 & 100 & 86.5 & 95.5 & 97.3 & 100 \\
\hline
\end{tabular}

3 
Table 4 (on next page)

Parameters of diagnostic deep myometrial invasion of PET/CT and Integrated PET/MRI on a per-patient basis 
1 Table 4 Parameters of diagnostic deep myometrial invasion of PET/CT and Integrated PET/MRI on a per2 patient basis

\begin{tabular}{lccc}
\hline & \multicolumn{3}{c}{ Deep myometrial invasion } \\
\cline { 2 - 4 } & overstaged & understaged & Accuracy \\
\hline PET/CT & 6 & 11 & $45.9 \%$ \\
Integrated PET/MRI & 3 & 5 & $81.8 \%$ \\
\hline
\end{tabular}

3 